\title{
Clinicopathologic Results of the Surgical Management of Thyroid Gland Pathologies
}

\author{
Original Investigation \\ Ali Cemal Yumuşakhuylu (D), Orhan Asya (D), Yavuz Gündoğdu (1), Çağatay Oysu \\ Department of Otolaryngology, Marmara University Pendik Training and Research Hospital, İstanbul, Turkey
}

\begin{abstract}
Objective: The aim of this study is to share our surgical approach and results in patients with benign and malignant thyroid diseases.

Methods: All patients who underwent thyroid gland surgery at our University Hospital Ear, Nose, and Throat Department between 2012 and 2017 were retrospectively analyzed. The study included 293 patients.

Results: Of the 293 patients included in the study, 76 (25.9\%) were male and 217 (74.1\%) were female. Mean follow-up period was 47.1 months. Patients' ages ranged from 4 to 77 years. In terms of the pathology, 160 patients had benign and 133 had neoplastic thyroid diseases. None of the patients who underwent thyroid surgery due to benign diseases required revi-
\end{abstract}

ORCID iDs of the authors: A.C.Y. $0000-0002-8421-211 X$; O.A. $0000-0003-0366-3099 ;$ Y.G. 0000-0003-3662-829X; ç.0. 0000-0002-6756-8456.

Cite this article as: Yumuşakhuylu AC, Asya 0 , Gündoğdu Y, Oysu Ç. Clinicopathologic Results of the Surgical Management of Thyroid Gland Pathologies. Turk Arch Otorhinolaryngol 2020; 58(2):93-8.

\section{Corresponding Author:}

Orhan Asya, orhan4913@gmail.com

Received Date: 09.03 .2020

Accepted Date: 19.05 .2020

Content of this journal is licensed under a Creative Commons Attribution 4.0 International License. Available online at www.turkarchotolaryngol.net

\section{Introduction}

Thyroidectomy is the treatment of choice for the majority of thyroid gland malignancies and a significant proportion of patients with benign thyroid diseases. Surgical planning is performed in regard of the patient's age, comorbid diseases, underlying thyroid gland disease, and the size of the disease. Currently, total thyroidectomy and loboisthmectomy are accepted as surgical approaches. Subtotal thyroidectomy and subtotal lobectomy, which have been performed frequently in the past, are no longer preferred due to increased recurrence rates (1). Total thyroidectomy is usually performed when surgical treatment is considered for both benign and malignant pathologies of the thyroid gland, whereas total loboisthmectomy is performed less frequently. Total thyroidectomy is required to eliminate the underlying pathology in patients with Graves' disease, a toxic multinodular goiter sion surgery. Recurrence occurred in 15 out of the 116 patients that were operated on for well-differentiated thyroid cancer. Out of these 15 patients with disease recurrence, 11 had biochemically incomplete responses and four had structurally recurrent diseases.

Conclusion: Thyroidectomy, when performed safely and correctly, is a very effective way of treating both benign and malignant thyroid diseases. Total thyroidectomy or total lobectomy and isthmectomy are the gold-standard surgical approaches to prevent recurrence. A national form is needed regarding thyroid surgery for following up on patients and for the regular and systematic collection of data.

Keywords: Thyroidectomy, fine-needle aspiration biopsy, thyroid cancer, thyroid surgery
(MNG), MNG causing compression symptoms and thyroid gland malignancies (2). In our clinic, the procedures performed in terms of thyroid gland surgery are total thyroidectomy or total loboisthmectomy. Subtotal thyroidectomy and subtotal loboisthmectomy are not performed.

\section{Methods}

All patients who underwent thyroid gland surgery between 2012 and 2017 at the Ear, Nose, and Throat Department of a tertiary medical center were retrospectively analyzed. The study was approved by the Marmara University Ethics Committee for Clinical Research on May 5, 2017 with number 09.2017.371. Informed consent was not obtained from patients because of the retrospective design of the study. The study is a descriptive research in which statistical analysis was done by simple calculating methods. The study included 
293 patients. Age and gender of the patients were noted and patients' family histories of thyroid cancer were documented. The preoperative evaluation of the patients included examining their thyroid gland and neck ultrasonography (USG), fine-needle aspiration biopsy (FNAB) results, vocal cord function evaluation, and thyroid scintigraphies. Likewise, in the postoperative period, we evaluated the specimen histopathologic examination results, complications, recurrence of the disease and the need for adjuvant therapy with radioactive iodine (RAI), chemotherapy, and radiotherapy of each patient. All patient data were obtained from our clinical archive for patients' pre- and post-surgery follow-up visits.

\section{Results}

Of the 293 patients included in the study, 76 (25.9\%) were male and $217(74.1 \%)$ were female. Patients' ages ranged from 4 to 77 years and the average age is 45.6. The mean follow-up period was 47.1 months. Familial papillary thyroid carcinoma was found in $5.3 \%$ (six of 114) of the cases. When the patients with neoplastic thyroid disease were evaluated in regard of their histopathological diagnosis, 114 of the 133 patients (85.7\%) with thyroid neoplasia had papillary thyroid carcinoma (PTC), four (3\%) had medullary thyroid carcinoma (MTC), two (1.5\%) had anaplastic thyroid carcinoma (ATC), two (1.5\%) had follicular thyroid carcinoma (FTC), one (0.75\%) had poorly differentiated cancer, one $(0.75 \%)$ had breast cancer thyroid metastasis, and nine (6.8\%) had follicular adenoma (FA) (Table 1). Types of benign thyroid disease in 160 patients and their FNAB results are given in Table 2 and 3 respectively. The FNAB results of 133 patients with thyroid malignancy are showed in Table 4.

Table 1. Postoperative histopathological diagnosis of thyroid neoplasms

\begin{tabular}{l|c|c}
\hline Histopathological diagnosis & Number of patients & $\%$ \\
\hline PTC & 114 & 85.7 \\
\hline MTC & 4 & 3 \\
\hline ATC & 2 & 1.5 \\
\hline FTC & 2 & 1.5 \\
\hline Poorly differentiated carcinoma & 1 & 0.75 \\
\hline Breast cancer thyroid metastasis & 1 & 0.75 \\
\hline FA & 9 & 6.8 \\
\hline
\end{tabular}

PTC: papillary thyroid carcinoma; MTC: medullary thyroid carcinoma; ATC: anaplastic thyroid carcinoma; FTC: follicular thyroid carcinoma; FA: follicular adenoma

\section{Main Points}

- Thyroidectomy is an effective treatment option that we can safely perform in patients with thyroid gland diseases.

- Patients with aggressive onset of disease and a high risk for recurrence should be followed closely and follow-up intervals should be more frequent than for other patients.

- Prophylactic lateral neck dissection without an FNAB should be avoided.

- A national form for the follow-up of patients with thyroid cancer and for the regular and systematic collection of data is needed.
The preoperative and postoperative vocal cord function of the patients were evaluated. Among patients with benign pathology, temporary unilateral vocal cord paralysis was seen in seven of 160 (4.4\%), while permanent unilateral vocal cord paralysis was seen in only two patients (1.2\%). Preoperative unilateral vocal cord paralysis was seen in three of the patients with benign pathology and none of these three patients showed improvement in their vocal cord motion after the operation. Transient and permanent unilateral vocal fold paralysis in patients with neoplastic thyroid disease was seen in 3.7\% (five of 133) and 3.7\% (five of 133) of the patients, respectively. There was unilateral vocal fold paralysis in two patients with neoplastic thyroid disease, and neither showed any change in the motion of their paralyzed vocal folds one year after the surgery.

Permanent hypocalcemia was seen in only one patient out of 293. Transient hypocalcemia was not evaluated in our study due to the lack of reliable data.

Table 2. Postoperative histopathological diagnosis of benign thyroid diseases

\begin{tabular}{l|c|c}
\hline Histopathological diagnosis & Number of patients & $\%$ \\
\hline MNG & 114 & 71.3 \\
\hline Graves' disease & 20 & 12.5 \\
\hline Solitary thyroid nodule & 17 & 10.6 \\
\hline Hashimoto thyroiditis & 9 & 5.6 \\
\hline MNG: multinodular goiter & &
\end{tabular}

Table 3. Fine needle aspiration biopsy findings of patients with benign thyroid pathology

\begin{tabular}{|c|c|c|c|}
\hline & & Number of patients & $\%$ \\
\hline \multirow[t]{7}{*}{ FNAB results } & Bethesda 1 & 7 & 4.4 \\
\hline & Bethesda 2 & 116 & 72.5 \\
\hline & Bethesda 3 & 9 & 5.6 \\
\hline & Bethesda 4 & 2 & 1.3 \\
\hline & Bethesda 5 & 4 & 2.5 \\
\hline & Bethesda 6 & 2 & 1.3 \\
\hline & FNAB not performed & 20 & 12.4 \\
\hline
\end{tabular}

FNAB: fine-needle aspiration biopsy

Table 4. Fine needle aspiration biopsy findings of patients with thyroid malignancy or follicular adenoma

\begin{tabular}{l|l|c|c}
\hline \multicolumn{2}{|c|}{} & Number of patients & $\%$ \\
\hline FNAB results & Bethesda 1 & 2 & 1.5 \\
\cline { 2 - 4 } & Bethesda 2 & 19 & 14.3 \\
\cline { 2 - 4 } & Bethesda 3 & 6 & 4.5 \\
\hline & Bethesda 4 & 13 & 9.7 \\
\hline & Bethesda 5 & 19 & 14.4 \\
\hline & Bethesda 6 & 74 & 55.6 \\
\hline
\end{tabular}

FNAB: fine-needle aspiration biopsy 
Table 5. Subgroups of well-differentiated thyroid carcinomas

\begin{tabular}{l|l|c|c}
\hline \multicolumn{2}{|l|}{} & Number of patients & $\%$ \\
\hline $\begin{array}{l}\text { Histopathological } \\
\text { diagnosis }\end{array}$ & Classical variant of PTC & 60 & 51.7 \\
\cline { 2 - 4 } & FVPTC & 32 & 27.6 \\
\cline { 2 - 4 } & $\begin{array}{l}\text { PTCs other than a } \\
\text { classical or follicular } \\
\text { variant }\end{array}$ & 22 & 19 \\
\cline { 2 - 4 } & FTC & 2 & 1.7 \\
\hline
\end{tabular}

PTC: papillary thyroid carcinoma; FVPTC: Follicular-variant of papillary thyroid carcinoma; FTC: follicular thyroid carcinoma

Table 6. Distribution of well-differentiated thyroid cancers according to the 2017 AJCC TNM classification

\begin{tabular}{l|c|c|c}
\hline \multirow{3}{*}{ Primary tumor } & & Number of patients & $\%$ \\
\cline { 2 - 4 } & T1a & 36 & 31 \\
\cline { 2 - 4 } & T1b & 44 & 37.9 \\
\cline { 2 - 4 } & T2 & 24 & 20.6 \\
\cline { 2 - 4 } & T3a & 9 & 7.8 \\
\hline Lymph node & T3b & 2 & 1.8 \\
\cline { 2 - 4 } & T4a & 1 & 0.9 \\
\cline { 2 - 4 } & Nx & 85 & 73.3 \\
\hline Metastasis & N1a & 9 & 7.8 \\
\hline \multirow{2}{*}{ Stage } & N1b & 14 & 6.9 \\
\hline & M0 & 114 & 12 \\
\hline
\end{tabular}

There were 116 patients with well-differentiated thyroid carcinoma (WDTC) and their sub-types in terms of histopathology are shown in Table 5.

Central neck dissection alone was performed in 11 of the $116 \mathrm{pa}^{-}$ tients. Both central and lateral neck dissection were performed in 20 of the 116 patients. Of the 20 patients who underwent lateral neck dissection, lymph node positivity was preoperatively proven by biopsy in 12 patients, whereas preoperative biopsy was not performed in eight patients. The decision to perform a neck dissection on these eight patients was made based on suspicious ultrasonographic and clinical findings, and only in two of the eight patients was lymph node positivity confirmed after the histopathologic examination of the lateral neck dissection specimens.

Well-differentiated thyroid cancer patients were evaluated according to the 2017 AJCC TNM classification: 80 patients had a T1 tumor; distant metastasis was seen in only two patients; $91.4 \%$ of the patients were Stage 1; and none were Stage 3 or 4 (Table 6).

Patients with WDTC were evaluated to discover whether they needed postoperative RAI, chemotherapy, or radiotherapy. Accordingly, $67.3 \%$ of the patients (78 of 116 ) received RAI, $1.7 \%$ (two of 116) received chemotherapy (sorafenib) for distant metastasis, and $0.9 \%$ (one of 116 ) received external beam radiotherapy for femur metastasis. Because RAI treatment was not available in our hospital, they were treated in other hospitals. So, their RAI treatment dosing regimens could not be presented.

Recurrence (structural disease or abnormal biochemical findings without detected structural disease) occurred in 16 of $116 \mathrm{pa}^{-}$ tients (13.8\%) with well-differentiated thyroid cancer and they received RAI or surgical treatment. None of the patients developed disease-related mortality.

Evaluation of tumor focus in well-differentiated thyroid cancers revealed that in 82 of 116 patients (70.7\%), the tumoral lesion was in a single lobe and unifocal, whereas in 34 patients (29.3\%), the tumor was multifocal or multicentric.

The follow-up periods of four patients with MTC were 29, 33, 53 , and 55 months, respectively. Two of these patients underwent total thyroidectomy, and bilateral central and bilateral functional neck dissections. The other two underwent total thyroidectomy and bilateral central neck dissection in their first operation and bilateral functional neck dissection in the follow-up period. Three received chemotherapy and radiotherapy in the follow-up period while one did not receive any therapy. All four patients were alive at the writing of the manuscript.

We had two cases of anaplastic thyroid cancer. One of them had a hard mass in the thyroid gland fixed in the surrounding tissues at the time of diagnosis. There was no systemic metastasis. Total thyroidectomy and bilateral central and functional neck dissections were performed together with a tracheotomy. Trachea and oesophagus invasions were observed during surgery. Disease-related mortality occurred five months after the surgery in this patient. The other patient had no neck metastasis. This patient underwent a total thyroidectomy, and trachea and oesophagus invasions were observed during surgery but tracheotomy was not performed. Disease-related mortality occurred two years after diagnosis in this patient.

There was one case of poorly differentiated thyroid cancer and total thyroidectomy was performed. One year after the operation, there was a recurrence in the left thyroid region and the left neck. Left functional neck dissection together with a mass excision in the left thyroid region were performed. This patient was followed for 50 months.

We had one case of invasive ductal breast cancer metastasis to the left thyroid lobe. This patient had undergone a left mastectomy and axillary lymph node dissection operation 12 years before the disease metastasized to the left thyroid lobe. A left thyroid lobectomy was done, and two years after the lobectomy, the patient died because of diffuse invasive ductal carcinoma metastasis.

\section{Discussion}

Surgical treatment is used effectively in benign and malignant thyroid gland diseases and can save patients from morbidity and 
mortality related to the disease. Thyroid gland cancers account for about $95 \%$ of all endocrine tumors and their incidence has increased steadily over the past two decades. In addition to this increase in incidence, cases of aggressive thyroid cancer have been increasing in the recent years, and mortality rates specific to thyroid cancer are gradually increasing. This increase in mortality occurs despite an early diagnosis and more effective treatment for thyroid cancer (3). Additionally, thyroid cancer is three times more common in women than in men (4). In our study, thyroid cancer was found to be 2.4 times more common in women - a finding consistent with the literature. Familial PTC is seen in about $5 \%$ of all thyroid cancer cases (5). In our study, this rate was found as $5.3 \%$, as also consistent with the literature.

In a study of 53,856 patients showing variations in thyroid cancer according to histological subtypes, the incidence of PTC increased while the incidence of FTC decreased. In the referred study, the incidence of PTC was 79\%, FTC was $13 \%$, and medullary thyroid cancer was $4 \%$ (6). However, when we examined the thyroid cancers according to histological subtypes, the incidence of PTC was found to be $92.7 \%$ (114 of 123) and FTC was found to be $1.6 \%$ (two of 123). Thus, the incidence of FTC was significantly lower than those reported in the literature. Follicular carcinoma accounts for $10 \%$ of all thyroid carcinomas (7). And, the incidence of follicular carcinoma is higher than $10 \%$ in areas with iodine deficiency. Addition of iodine to the diet results in relative increase in papillary carcinoma and corresponding decrease in follicular carcinoma (8). The relatively low incidence of follicular carcinoma in our series may be related to sufficient iodine supplementation in our country.

Thyroid cancer or FA was detected in $14.3 \%$ of patients (19 of 133) whose FNAB was reported as benign cytology. Of these 19 patients, 15 were diagnosed with PTC, one with FTC, and three with FA. As a result, malignancy was detected in $12 \%$ of the patients (16 of 133) whose FNAB was reported as benign. The 2015 ATA management guideline for adult patients with differentiated thyroid cancer, states that $1-4 \%$ of instances of FNAB benign cytology may become malignant (9). In a meta-analysis, the probability of thyroid cancer in cases reported as benign cytology with an FNAB was determined to be $1 \%$ to $10 \%(10)$. In studies where the probability of malignancy is higher in cases with benign cytology, it was seen that a biopsy of palpable nodules was usually not performed with ultrasound guidance (USG) and this rate was lower in USG biopsies. This reveals the importance of USG biopsies, even if the nodule is palpable. The 16 cases with Bethesda 2 cytology results in whom the thyroidectomy specimen was reported as thyroid cancer in our series were further examined individually for the cause of false cytological negativity. Incidental papillary microcarcinoma was detected in eight of these 16 patients, and the nodule that was aspirated for cytology was not the source of the malignant nodule. Thus, the FNAB was reported as benign cytology in $6 \%$ of the cases (eight of 133), even though an aspirate was taken from the malignant nodule. Rarely, the FNAB of palpable nodules is not performed under USG in our clinic and the high rate of $6 \%$ can be attributed to this con- dition. However, we do not know in which patients' biopsies were taken under USG. Furthermore, FNABs were examined by different pathologists, whereas examination of all thyroid FNABs by a single qualified pathologist may result in a reduction in this high percentage.

None of our patients had permanent or transient bilateral vocal cord paralysis as a surgical complication. The incidence of postoperative transient vocal cord paralysis was $4 \%$ (12 of 293) while that of permanent vocal cord paralysis was $2.4 \%$ (seven of 293). These rates are similar to those in the literature. In a retrospective study of 119 patients who underwent thyroid surgery, vocal cord paralysis was found in 6.7\% (eight of 119) of the patients (11).

In our study, 12 of the 20 patients who underwent lateral neck dissection had preoperative biopsy-proven lateral neck lymph node metastasis and, in eight patients, lateral neck dissection was performed without preoperative biopsy based on suspicious malignant sonographic and clinical findings. Of these eight patients, only two had lymph node metastasis as proven by the histopathological examination of neck dissection specimens. The 2015 American Thyroid Association Management Guidelines for Adult Patients with Thyroid Nodules and Differentiated Thyroid Cancer recommendation 37 states that lateral neck dissection should be performed in patients with biopsy-proven lymph node metastasis in the lateral neck. The sonographic findings, palpable lateral neck lymph nodes, and primary tumor size are important when deciding to perform a lymph node FNAB. However, a prophylactic lateral neck dissection without FNAB may be unnecessary in many patients, prolonging their operation time and causing morbidity. Given the fact that only two out of eight cases with suspicious lateral lymph nodes were found to be metastatic, we no longer practice and recommend elective neck dissection for suspicious lymph nodes. FNAB and thyroglobulin wash out of suspicious lymph nodes should be performed, and lateral neck dissection should only be done therapeutically when either result is found to be positive.

None of the patients who underwent thyroid surgery for a benign disease required revision surgery. This can be because the recurrent laryngeal nerve was identified during surgery and either total thyroidectomy or total lobectomy and isthmectomy was performed in all patients undergoing thyroidectomy.

Patients with ATC may present with intrathyroidal mass in $10 \%$ of cases, extrathyroidal invasion and/or lymph node metastasis in $40 \%$ of cases and widely metastatic disease in $50 \%$ of cases $(12,13)$. Total thyroidectomy and therapeutic central and lateral lymph node dissection should be considered in patients with resectable disease. Complete resection of the tumor or the microscopic residual tumor is associated with improved disease-free survival and overall survival. In patients with extrathyroidal invasion, an en bloc resection with the goal of achieving gross negative margins should be considered. Incomplete resection or tumor debulking should not be performed (14). One of the two ATC patients in our series had intrathyroidal mass without metastatic lymph nodes or distant metastasis. Total thyroidectomy 
with microscopic residual tumor was done and the patient had disease related mortality two years after the surgery. The second patient who had firm thyroid mass with extrathyroidal invasion underwent surgery with the goal of achieving gross negative margins. Total thyroidectomy, bilateral central and lateral neck dissections together with tracheotomy were performed. The goal of grossly negative margins was achieved but unfortunately the patient had disease related mortality five months after the surgery.

Recurrence occurred in 15 out of the 116 patients with well-differentiated thyroid cancer. Eleven of them had a high serum thyroglobulin level and locoregional RAI uptake in the neck during the first three years of the postoperative period. These patients received RAI treatment, from which they benefited. There was no evidence of a structurally recurrent disease in these 11 patients. The other four patients who developed recurrence had a poor onset and worse course of the disease. In these four patients, there was locoregional or metastatic structurally recurrent disease. One of these patients presented with stridor and hoarseness. Neck mass and right vocal cord paralysis were observed in the examination of the patient. The patient had difficulty in breathing. Chest radiography revealed bilateral multiple opacity with suspicion of malignancy. There were multiple millimetric bilateral multifocal lung metastases. PTC was detected in subsequent examinations. A total thyroidectomy, as well as bilateral central and lateral neck dissections together with a tracheotomy were performed. Tracheotomy was performed because of stridor, hoarseness, and multiple lung metastases. The histopathologic examination was reported as classical variant of PTC and he received postoperative RAI treatment and oral sorafenib for four weeks. The patient was decannulated in the fifth postoperative month. In the first postoperative year, structurally recurrent disease occurred in the bilateral neck and the patient underwent revision lateral neck dissection bilaterally. This patient received RAI treatment four times in total and has been followed up for six years. There is no evidence of a structural disease as yet. And currently, both vocal cords are mobile. The second patient had a tumor size of eight $\mathrm{cm}$ in the right thyroid lobe as well as neck metastasis causing right internal jugular vein invasion. A total thyroidectomy, and bilateral central and lateral neck dissections were performed and the internal jugular vein was ligated. In this patient, whose pathology was reported as an oncocytic variant of PTC, a structurally recurrent disease developed on the right side of the neck twice after the first operation and the patient underwent a revision right neck dissection. A total of three RAI treatments were administered to the patient. The patient has been followed up for four years and there is no evidence of a structural disease but there is a biochemically incomplete response. The third patient had a tumor of $4.3 \mathrm{~cm}$ in size and underwent a total thyroidectomy. Diffuse vascular invasion was detected in this patient whose histopathologic examination was reported as an oncocytic variant of PTC. The patient was given RAI treatment. Two years after the operation, the patient developed liver metastasis. The patient was given transarterial radioembolization with yitrium-90 for the liver metastasis after which a segmental liver resection was performed. The patient has been followed up for 3.5 years and there is widespread liver and bone metastases. In the fourth patient who developed structural recurrence, papillary cancer vertebra metastasis was diagnosed preoperatively and a total thyroidectomy and bilateral central and lateral neck dissections were performed. No lymph node metastasis was detected in the patient whose histopathologic examination was reported as FTC. This patient received 200 millicuries of RAI as well as RT for the vertebral metastasis. One year after the operation, both vertebrae and femur metastases developed. Partial left femur resection with osteosynthesis was done. The patient was given radiotherapy for the vertebra again. This patient has been followed up for two years and there is a biochemically incomplete response now without evidence of a structural disease as yet.

\section{Conclusion}

A FNAB of thyroid nodules should be performed under USG as these biopsies have higher diagnostic accuracy. USG is necessary for aspiration from the most suspected malignant portion of the nodule, even if the nodule is palpable. Suspicious sonographic findings, palpable lateral neck lymph nodes, and a large primary tumor size are valuable inputs when deciding to perform a lymph node FNAB, but a prophylactic lateral neck dissection without an FNAB should be avoided. Without biopsy-proven lateral neck metastasis, lateral neck dissections unnecessarily prolong the operation time and cause morbidity in many patients. It is known that the disease has a more aggressive course in thyroid cancer patients who experience an aggressive onset of the disease. In this context, cases with aggressive onset and a high risk for recurrence should be followed closely and follow-up intervals should be more frequent than for other patients. In addition, they should receive RAI treatment in the earliest possible postoperative period. However, we do not have a national form for the follow-up of patients with thyroid cancer and for the regular and systematic collection of data.

Ethics Committee Approval: Ethics committee approval was received for this study from the Ethics Committee for Clinical Research of Marmara University School of Medicine (Approval Date: May 5, 2017; Approval Number: 09.2017.371).

Informed Consent: Written informed consent was not obtained due to the retrospective nature of the study.

Peer-review: Externally peer-reviewed.

Author Contributions: Concept - A.C.Y., O.A., Y.G., Ç.O.; Design - A.C.Y., O.A., Y.G., Ç.O.; Supervision - A.C.Y., O.A., Y.G., Ç.O.; Materials - O.A., Y.G.; Data Collection and/or Processing - O.A., Y.G.; Analysis and/or Interpretation - A.C.Y., O.A., Y.G., Ç.O.; Literature Search - O.A., Y.G.; Writing - A.C.Y., O.A., Y.G., Ç.O.; Critical Reviews - A.C.Y., O.A., Y.G., Ç.O.

Conflict of Interest: The authors have no conflicts of interest to declare.

Financial Disclosure: The authors declared that this study has received no financial support. 


\section{References}

1. Röjdmark J,Järhult J. High long term recurrence rate after subtotal thyroidectomy for nodular goitre. EurJ Surg 1995; 161: 725-7.

2. Patel UA. Surgical treatment of thyroid disease. In: Washington Manual Otolaryngology Survival Guide. Lippincott Williams \& Wilkins, 2003.

3. Pellegriti G, Frasca F, Regalbuto C, Squatrito S, Vigneri R. Worldwide increasing incidence of thyroid cancer: update on epidemiology and risk factors. J Cancer Epidemiol 2013; 2013: 965212. [Crossref]

4. Rahbari R, Zhang L, Kebebew E. Thyroid cancer gender disparity. Future Oncol 2010; 6: 1771-9. [Crossref]

5. Malchoff CD, Malchoff DM. Familial nonmedullary thyroid carcinoma. Cancer Control. 2006;13:106-10. [Crossref]

6. Hundahl SA, Fleming ID, Fremgen AM, Menck HR. A national cancer data base report on 53,856 cases of thyroid carcinoma treated in the U.S., 1985-1995. Cancer 1998; 83: 2638-48. [Crossref]

7. Hundahl SA, Cady B, Cunningham MP, Mazzaferri E, McKee RF, Rosai J, et al. Initial results from a prospective cohort study of 5583 cases of thyroid carcinoma treated in the United States during 1996: an American college of surgeons commission on cancer patient care evaluation study. Cancer 2000; 89: 202-17. [Crossref]

8. Rosai J, Carangui ML, DeLellis RA. Atlas of tumor pathology: tumors of the thyroid gland. Washington, DC: Armed Forces Institute of Pathology; 1992.
9. Haugen BR, Alexander EK, Bible KC, Doherty GM, Mandel SJ, Nikiforov YE, et al. 2015 American Thyroid Association management guidelines for adult patients with thyroid nodules and differentiated thyroid cancer: the American Thyroid Association guidelines task force on thyroid nodules and differentiated thyroid cancer. Thyroid. 2016; 26: 1-133. [Crossref]

10. Bongiovanni M, Spitale A, Faquin WC, Mazzucchelli L, Baloch ZW. The Bethesda system for reporting thyroid cytopathology: a meta-analysis. Acta Cytol 2012; 56: 333-9. [Crossref]

11. Paksoy M, Aydın S, Ayduran E, Eken M, Şanlı A, Taşdemir Ö. Tiroid kitlelerinde klinik bulgular ve uyguladığımız tedavi yöntemleri. Kulak Burun Boğaz İhtis Derg 2008; 18: 294-9.

12. McIver B, Hay ID, Giuffrida DF, Dvorak CE, Grant CS, Thompson GB, et al. Anaplastic thyroid carcinoma: a 50-year experience at a single institution. Surgery 2001; 130: 1028-34. [Crossref]

13. Chen J, Tward JD, Shrieve DC, Hitchcock YJ. Surgery and radiotherapy improves survival in patients with anaplastic thyroid carcinoma: analysis of the surveillance, epidemiology, and end results 1983-2002. Am J Clin Oncol 2008; 31: 460-4. [Crossref]

14. Smallridge RC, Ain KB, Asa SL, Bible KC, Brierley JD, Burman $\mathrm{KD}$, et al. American Thyroid Association guidelines for management of patients with anaplastic thyroid cancer. Thyroid 2012; 22: 1104-39. [Crossref] 